\title{
A RELATIONSHIP BETWEEN THE CURVATURE TENSOR AND THE DIFFERENCE TENSOR FOR AFFINE HYPERSURFACES
}

\author{
Daniel A. Joaquín ${ }^{1 *}$ \\ *l Departamento de Matemática, Fac. de Cs. Exs., Fis. y Nat., Universidad Nacional de Córdoba Avda. Velez Sarsfield \\ 299, 5000 Córdoba (ARGENTINA) e-mail:dajoaquin@yahoo.com.ar
}

\section{*Corresponding Author: -}

Email: dajoaquin@yahoo.com.ar

\begin{abstract}
: -
In the present work, it is obtained a class of hypersurfaces, of decomposable type, for which the curvature tensor associated to the affine normal connection, and the Levi-Civita covariant derivative of the difference tensor, are scalar multiples each other.
\end{abstract}

\section{(c) (\$) (1)}




\section{INTRODUCTION}

Let $M$ be an $n$-dimensional $C^{\infty}$ manifold, and $F: M \rightarrow \mathrm{R}^{n+1}$ an immersion of class $C^{\infty}$. Consider the affine space $\mathrm{R}^{n+1}$ with its usual flat connection $D$ and a volume form $\omega$.

If we denote $\xi$ the affine normal field and $\nabla$ the induced connection ( called normal connection)and $S$ is the affine shape operator, we have the formula of Gauss

$$
D_{X} Y=\nabla_{X} Y+h(X, Y)
$$

and the formula of Weingarten

$$
D_{X} \xi=-S X
$$

The curvature tensor field $R$, which is of type $(1,3)$ is defined by

$$
R(X, Y) Z=\nabla_{X} \nabla_{Y} Z-\nabla_{Y} \nabla_{X} Z-\nabla_{[X, Y]} Z
$$

and the torsion tensor field $T$ is defined by

$$
T(X, Y)=\nabla_{X} Y-\nabla_{Y} X-[X, Y]
$$

If we denote $\widetilde{\nabla}$ the Levi-Civita connection for $h$, we can consider the difference tensor field $K$, which is of type $(1,2)$, given by the diference between the normal and Levi-Civita connections, that is

$$
K(X, Y)=\nabla_{X} Y-\widetilde{\nabla}_{X} Y
$$

Because of $\nabla$ and $\tilde{\nabla}$ they are both torsion-free, we have $K(X, Y)=K(Y, X)$, and usually we write $K_{X}=\nabla_{X}-\widetilde{\nabla}_{X}$. The covariant differentiation of $K$, with respect to Levi - Civita connection gives a tensor field

$\widetilde{\nabla} K$, wuich is of $(1,3)$ type, and we ask ourselves if it feasible to have the equation $\widetilde{\nabla} K=\lambda R$ fulfilled (where $\lambda$ is a scalar, possibily dependent on the dimension of $M$ ) for some hypersurfaces decomposable type.

\section{Geomety of affine immersions}

Let $M$ be an $n$-dimensional manifold of class $C^{\infty}$ and let $F: M \rightarrow \mathrm{R}^{n+1}$ be an affine immersion of class $C^{\infty}$. If $\xi$ denotes the affine normal field and $(\nabla, h, S)$ is the Blaschke structure on the hypersurface $M$, we have the fundamental equations

$$
\begin{array}{crc}
R(X, Y) Z=h(Y, Z) S X-h(X, Z) S Y \quad \text { Gauss equation } & 2.1 \\
\left(\nabla_{X} h\right)(Y, Z)=\left(\nabla_{Y} h\right)(X, Z) & \text { Codazzi equation for } h & 2.2 \\
\left(\nabla_{X} S\right) Y=\left(\nabla_{Y} S\right) X & \text { Codazzi equation for } S & 2.3 \\
h(S X, Y)=h(X, S Y) & \text { Ricci equation } & 2.4
\end{array}
$$

2.5 Definition. The Ricci tensor field Ric, which is of $(0,2)$ type, is given by

$$
\operatorname{Ric}(X, Y)=\operatorname{trace}\{Z \rightarrow R(Z, X) Y\}
$$

2.6 Proposition. The Riccitensor is given by $\operatorname{Ric}(X, Y)=\operatorname{Tr}(S) h(Y, Z)-h(S Y, Z)$ and Ric=0 if, and only if $S=0$.

Proof. [1]. Proposition 3.4, page 42.

Now, we define two more tensors, from $\nabla, \widetilde{\nabla}$, and $K$.

$$
\widetilde{L}(X, Y)=\operatorname{trace}\left\{Z \rightarrow\left(\widetilde{\nabla}_{Z} K\right)(X, Y)\right\}
$$

and the analogous for the normal connection $\nabla$

$$
L(X, Y)=\operatorname{trace}\left\{Z \rightarrow\left(\nabla_{Z} K\right)(X, Y)\right\}
$$

\subsection{Proposition.}

$$
\begin{gathered}
\widetilde{L}(X, Y)=\frac{1}{2} \operatorname{Tr}(S) h(X, Y)-\frac{n}{2} h(S X, Y) \\
\widetilde{L}(X, Y)-L(X, Y)=2 \operatorname{Tr}\left(K_{X} K_{Y}\right)
\end{gathered}
$$

Proof. [1]. Propositions 9.4 y 9.9, pages 79 y 82 respectively.

From 2.10 we see immedialy that a hypersurface is an affine hypersphere $(S=\mu I)$ if, and only if $\tilde{L}=0$. 
2.12. Proposition. If $\widetilde{\nabla} K=\lambda_{R}$, then $\widetilde{L}=0$, that is, the hypersurface is an affine hypersphere.

Proof. $\tilde{\nabla} K=\lambda_{R \text { implies }}\left(\tilde{\nabla}_{Z} K\right)(X, Y)=\lambda R(X, Y) Z$, then

by using (2.1) and (2.4).

$$
\begin{aligned}
\widetilde{L}(X, Y) & =\operatorname{trace}\left\{Z \rightarrow\left(\widetilde{\nabla}_{Z} K\right)(X, Y)\right\} \\
& =\operatorname{trace}\{Z \rightarrow \lambda R(X, Y) Z\} \\
& =\operatorname{trace}\{Z \rightarrow \lambda h(Y, Z) S X-\lambda h(X, Z) S Y\} \\
& =\lambda h(Y, S X)-\lambda h(X, S Y) \\
& =0
\end{aligned}
$$

2.13. Corollary. If $\widetilde{\nabla} K=\lambda R$, then $L(X, Y)=-2 \operatorname{Tr}\left(K_{X} K_{Y}\right)$.

Proof. Is immediate, for (2.11).

\section{Hypersurfaces Of Decomposable Type}

We now add the hypothesis that the hypersurface can be expressed with respect to a suitable affine coordinate system by

$$
F\left(t_{1}, \ldots, t_{n}\right)=\left(t_{1}, \ldots, t_{n}, f\left(t_{1}, \ldots, t_{n}\right)\right)
$$

with $\left(t_{1}, \ldots, t_{n}\right)$ varying in an open connected subset of $\mathrm{R}^{n}$ and $f$ can be decomposed into a sum of $n$ terms, each of them depending on only one of the independent variables $t_{1}, \ldots, t_{n}$, i.e.

$$
f\left(t_{1}, \ldots, t_{n}\right)=f_{1}\left(t_{1}\right)+f_{2}\left(t_{2}\right)+\cdots+f_{n}\left(t_{n}\right)
$$

The tensor field $h$ of unimodular affine geometry, in terms of the graph function $f$, is

$$
h=\sum_{i, j} h_{i j} d t_{i} \otimes d t_{j}
$$

Where

$$
h_{i j}=\delta_{i j} \phi^{-1} f_{i}^{\prime \prime}
$$

and

$$
\phi=\left(\prod_{k}\left|f_{k}^{\prime \prime}\right|\right)^{\frac{1}{n+2}}
$$

If $\left(h^{i j}\right)$ denotes the inverse matrix of $\left(h_{i j}\right)$ the Christoffel symbols $\widetilde{\Gamma}_{i j}^{k}$ of Levi-Civita connection are given by

$$
\widetilde{\Gamma}_{i j}^{k}=\frac{1}{2} \sum_{m} h^{k m}\left(\partial_{i} h_{m j}+\partial_{j} h_{i m}-\partial_{m} h_{i j}\right)
$$

from which

$$
\begin{gathered}
\widetilde{\Gamma}_{i i}^{i}=\frac{n+1}{2(n+2)} \frac{f_{i}^{\prime \prime \prime}}{f_{i}^{\prime \prime}} \\
\widetilde{\Gamma}_{i j}^{i}=\frac{1}{2(n+2)} f_{j}^{\prime \prime} \frac{f_{i}^{\prime \prime}}{\left(f_{i}^{\prime \prime}\right)^{2}} \quad i \neq j \\
\widetilde{\Gamma}_{i j}^{i}=\frac{-1}{2(n+2)} \frac{f_{i}^{\prime \prime}}{f_{i}^{\prime \prime}} \quad i \neq j \\
\widetilde{\Gamma}_{i j}^{k}=0 \quad i \neq j \neq k \neq i
\end{gathered}
$$

It can be seen, [2], that the Christoffel symbols $\widetilde{\Gamma}_{i j}^{k}$ of normal connection are given by

$$
\Gamma_{i j}^{k}=h_{i j} \sum_{m} h^{k m} \partial_{m} \log (\phi)
$$

from which 


$$
\begin{gathered}
\Gamma_{i i}^{i}=\frac{1}{n+2} \frac{f_{i}^{\prime \prime \prime}}{f_{i}^{\prime \prime}} \\
\Gamma_{j j}^{i}=\frac{1}{n+2} f_{j}^{\prime \prime} \frac{f_{i}^{\prime \prime}}{\left(f_{i}^{\prime \prime}\right)^{2}} \quad i \neq j \\
\Gamma_{i j}^{k}=0 \quad \text { otherwise }
\end{gathered}
$$

With the Christoffel symbols of both connections, we calculate the coefficients ${ }_{j k}^{i}$ of the tensor difference $K$

$$
\begin{gathered}
K_{i i}^{i}=\frac{1-n}{2(n+2)} \frac{f_{i}^{\prime \prime}}{f_{i}^{\prime \prime}} \\
K_{i j}^{i}=\frac{1}{2(n+2)} \frac{f_{j}^{\prime \prime}}{f_{j}^{\prime \prime}} \quad i \neq j \\
K_{j j}^{i}=\frac{1}{2(n+2)} f_{j}^{\prime \prime} \frac{f_{i}^{\prime \prime}}{\left(f_{i}^{\prime \prime}\right)^{2}} \quad i \neq j \\
K_{j k}^{i}=0 \quad \text { otherwise }
\end{gathered}
$$

The coefficients $S_{j}^{i}$ are given by

$$
\begin{aligned}
& S_{i}^{i}=\frac{\phi}{(n+2)^{2}}\left((n+2) h_{i}-(2 n+3) g_{i}\right) \\
& S_{j}^{i}=\frac{\phi}{(n+2)^{2}} \frac{f_{i}^{\prime \prime \prime} f_{j}^{\prime \prime \prime}}{\left(f_{i}^{\prime \prime}\right)^{2} f_{j}^{\prime \prime}} \quad i \neq j \\
& \text { Where } g_{k}=\frac{\left(f_{k}^{\prime \prime \prime}\right)^{2}}{\left(f_{k}^{\prime \prime}\right)^{3}} \text { and } h_{k}=\frac{f_{k}^{(4)}}{\left(f_{k}^{\prime \prime}\right)^{2}} \text {. }
\end{aligned}
$$

The derivatives $g_{k}^{\prime}$ and $h_{k}^{\prime}$ satisfies the following relations [3]

$$
\begin{gathered}
g_{k}^{\prime}=\frac{f_{k}^{\prime \prime}}{f_{k}^{\prime \prime}}\left(2 h_{k}-3 g_{k}\right) \\
h_{k}^{\prime}=\frac{1}{\left(f_{k}^{\prime \prime}\right)^{2}}\left(f_{k}^{(5)}-2 f_{k}^{\prime \prime} f_{k}^{\prime \prime \prime} h_{k}\right)
\end{gathered}
$$

Using (3.15), (3.16) and the Gauss equation (2.19), we compute the component $R_{j k l}^{i}$ of the tensor $R$ as

$$
R_{j k l}^{i}=h_{k l} S_{j}^{i}-h_{j l} S_{k}^{i}
$$

\section{Covariant Differentiation of the Difference Tensor Multiple of Curvature Tensor.}

This multiplicity is given by $\widetilde{\nabla} K=\lambda R$, that in coordinates is equivalent to $K_{j k, l}^{i}=\lambda R_{j k l}^{i}$, where the components $K_{j k, l}^{i}$ are given by

$$
K_{j k, l}^{i}=\partial_{l} K_{j k}^{i}+\sum_{m} \widetilde{\Gamma}_{l m}^{i} K_{j k}^{m}-\sum_{m} \widetilde{\Gamma}_{l j}^{m} K_{m k}^{i}-\sum_{m} \widetilde{\Gamma}_{l k}^{m} K_{j m}^{i}
$$

We establish the following convention for the indices: for different symbols, correspond different values too, and they range from 1 to $n$. Hence, the equations that we consider are:

$$
\begin{gathered}
\frac{n-1}{4(n+2)}\left[(3 n+5) g_{i}-2(n+2) h_{i}\right]-\frac{3}{4(n+1)^{2}} \sum_{m \neq i} g_{m}=0 \\
f_{i}^{\prime \prime \prime} f_{j}^{\prime \prime \prime}=0 \\
2(n+2) h_{i}-(3 n+5) g_{i}+(n+1) g_{j}-\sum_{m \neq i} g_{m}=0 \\
(n-1) g_{i}+2(n+2) h_{j}-(3 n+5) g_{j}+\sum_{m \neq i} g_{m}=\lambda\left[4(n+2) h_{i}-4(2 n+3) g_{i}\right]
\end{gathered}
$$

Remark: There are more equations, but they are omitted since they are redundant. 
From (4.3) we see that at least $n$-1 functions must be of parabolic type. Denoting $f_{k 0}$ the remaining one and assuming that $f_{k_{0}}^{\prime \prime \prime} \neq 0$. Making $i=k_{0}$ in (4.2), (4.4) and (4.5) we have

$$
\begin{aligned}
2(n+2) h_{k_{0}}-(3 n+5) g_{k_{0}} & =0 \\
(n-1) g_{k_{0}} & =4 \lambda\left[(n+2) h_{k_{0}}-4(2 n+3) g_{k_{0}}\right]
\end{aligned}
$$

and reordering

$$
\begin{array}{r}
(3 n+5) g_{k_{0}}-2(n+2) h_{k_{0}}=0 \\
(n-1+12 \lambda+8 \lambda n) g_{k_{0}}-4 \lambda(n+2) h_{k_{0}}=0
\end{array}
$$

and since $g_{k_{0}} \neq 0$, it must hold

$$
\operatorname{det}\left[\begin{array}{cc}
3 n+5 & -2(n+2) \\
n-1+12 \lambda+8 \lambda n & -4 \lambda(n+2)
\end{array}\right]=0
$$

that is, $2(n+2)(2 \lambda(n+1)+n-1)=0$, from which $\lambda=\frac{1-n}{2(n+1)}$ and replacing in (4.7) we have

Hence,

$$
(3 n+5) g_{k_{0}}-2(n+2) h_{k_{0}}=0
$$

or equivalently

$$
h_{k_{0}}=\frac{3 n+5}{2(n+2)} g_{k_{0}}
$$

$$
\frac{f_{k_{0}}^{(4)}}{\left(f_{k_{0}}^{\prime \prime}\right)^{2}}=\left(\frac{3 n+5}{2(n+2)}\right) \frac{\left(f_{k_{0}}^{\prime \prime}\right)^{2}}{\left(f_{k_{0}}^{\prime \prime}\right)^{3}}
$$

which solution, normalizing constants, is

$$
f_{k_{0}}\left(t_{k_{0}}\right)=t_{k_{0}}^{-\frac{2}{n+1}}, \quad t_{k_{0}}>0
$$

Of course, the $n$ functions there can not be of parabolic type, since in this case, the tensors $K$ and $R$ vanish. We resume all this in the following theorem:

4.9 Theorem. Let $M$ be a hypersurface of decomposable type with parametrization given by

$$
\left(t_{1}, \ldots, t_{n}\right) \rightarrow\left(t_{1}, \ldots, t_{n}, f_{1}\left(t_{1}\right)+\cdots+f_{n}\left(t_{n}\right)\right)
$$

Then, the condition $\widetilde{\nabla} K=\lambda R$ hold, if $\lambda=\frac{1-n}{2(n+1)}, n-1$ of functions $f_{k}$ are of parabolic type and the remaining one is given by $t \rightarrow t^{-\frac{2}{n+1}}$.

\section{References}

[1].K. Nomizu and T. Sasaki, Affine Differential Geometry, Cambridge University Press, (1994).

[2].D. Joaquín, Manifolds with Integrable Affine Shape Operator, Le Matematiche, Vol. LX (2005) - Fasc. 1, pp. 13 - 27. [3].S. Gigena, D. Joaquín, On affine hypersurfaces with constant sectional curvature. Math.

Notae. (1997/98), 63-81. 